\title{
Headache with neurological deficits and CSF lymphocytosis: a meningism and psychosis mimic
}

\author{
Akhila Kumar Panda, Muralikrishnan K, Garima Sarraf, Seema Mallik \\ Department of Neurology, Institute of Human Behaviour and Allied Sciences (IHBAS), Delhi, India
}

Email address:

drakhilapanda@gmail.com(A. K. Panda),drkmuralikrishnan@gmail.com(Muralikrishnan K), garimasarraf77@gmail.com(G. Sarraf), docmalik_01@hotmail.com(S. Mallik)

To cite this article:

Akhila Kumar Panda, Muralikrishnan K, Garima Sarraf, Seema Mallik. Headache with Neurological Deficits and CSF Lymphocytosis: A Meningism and Psychosis Mimic. American Journal of Internal Medicine Vol. 1, No. 1, 2013, pp. 7-9. doi: 10.11648/j.ajim.20130101.12

\begin{abstract}
Headache with neurological deficits and cerebrospinal fluid (CSF) lymphocytosis (HaNDL), a benign condition, known to have confused physicians and neurologists presenting like transient Ischemic Attacks (TIA), central nervous system infections and encephalitis of various etiology. HaNDL is typically seen in young adults, manifested as a triad of headache, transient focal neurological deficits and CSF lymphocytosis. The authors report the case of a 50 -year- old man with episodic headache associated with neck stiffness, altered sensorium and symptoms of acute psychosis. After extensive evaluation including neuroimaging, CSF study, different blood investigations to exclude possible central nervous system infections, autoimmune encephalitis and CNS vascular disease, a diagnosis of HaNDL was arrived. The patient improved with supportive and symptomatic management. HaNDL can present with symptoms akin to meningitis and psychosis. Due caution should be exercised before arrival at the diagnosis of HaNDL, which is a based on exclusion of other life threatening neurologic illness.
\end{abstract}

Keywords: Pseudo migraine, Meningism, Psychosis, CSF, MRI

\section{Introduction}

It is not uncommon to have young males brought in with history of altered sensorium associated with headache. Such scenarios warrant emergent extensive evaluation to rule out potentially fatal and disabling conditions such as cerebrovascular accidents, infectious intracranial pathology. It is ironic that though one may suspect an entity like Headache with neurological deficits and cerebrospinal fluid (CSF) lymphocytosis (HaNDL), which is virtually impossible to avoid expensive investigations that rule out the spectrum of fatal diseases that the HaNDL may mimic.

HaNDL is a benign, self limiting and self resolving condition characterized by headaches, episodic focal neurological deficits and lymphocytic pleocytosis, described in recent past[1-4]. HaNDL; though has commonly been reported to mimic TIA, very rarely has it presented with acute confusional state as its core feature.[5-8]. HaNDL has also been reported to have the risk of the neurological deficits getting precipitated post magnetic resonance angiography (MRA)[1-4].

We would like to report an extremely rare presentation of HaNDL, presenting with symptoms mimicking those of meningitis and acute psychosis.

\section{Case Report}

The neurology services at our hospital evaluated a 50 year old male, daily wage worker, with no history of preceding neurologic illness with complaints of severe continuous, bi-frontal, pulsating headache associated with vomiting. $\mathrm{He}$ was conscious, oriented until the intensity of headache increased, when he developed restless behavior and was unable to recognize his family members. The headache was neither preceded by fever, vomiting, aura, photophobia, phonophobia, conjuctival congestion nor associated with any focal neurologic deficit. Following headache, he was disoriented, not recognizing family member, shouting irrelevantly, became violent, throwing objects, abusive to family members who persisted for 24 hour. The symptoms resolved spontaneously without any definitive management. There was a similar episode four years back, when he was admitted at another hospital and a lumbar puncture was done, of which no records were available. There was neither similar headache in between, nor family history of migraine. At admission, general examination was unremarkable. 
Neurological assessment showed patient was disoriented, aggressive, with positive meningeal sign (neck stiffness and Kernig's sign).Cranial nerve examinations were grossly normal with normal motor system examinations including bilateral flexor plantar response. Sensory examination was grossly normal as he can perceive pain and temperature sensation. Keeping in mind the possibility of CNS infections like meningitis or encephalitis, CSF was subjected.CSF analysis showed the following: cell counts was 48 cells (all lymphocytes), sugar was $51 \mathrm{mg} \%$, protein was $97 \mathrm{mg} / \mathrm{dl}$, chloride $-135 \mathrm{mEq} / \mathrm{L}$. India ink, Gram's and acid fact staining were negative. CSF cultures for tuberculosis, pyogenic and fungal organisms were negative. PCR for tuberculosis and herpes simplex virus were negative. Magnetic resonance imaging (MRI) of brain with gadolinium contrast was noncontributory. Electroencephalogram (EEG) was normal. There was no evidence of extra pulmonary tuberculosis. He was diagnosed as probable tubercular meningitis in view of headache, altered sensorium, positive meningeal sign, CSF study and high prevalence of tuberculosis in the community. He was started on anti-tubercular therapy( Isoniazide $5 \mathrm{mg} / \mathrm{kg}$, rifampicin $10 \mathrm{mg} / \mathrm{kg}$, ethambutol $20 \mathrm{mg} / \mathrm{kg}$ and pyrazinamide $30 \mathrm{mg} / \mathrm{kg}$ ) and oral prednisolone of $1 \mathrm{mg} / \mathrm{kg}$ for one month followed by tapering over next one month.

After a symptom free period of 81 days, the patient presented with same complaints such as headache and acute psychosis where psychosis was present for $12 \mathrm{hr}$. Isoniazid was stopped and a CSF study was repeated, revealing 10 cells (all lymphocytes), protein $-42 \mathrm{mg} \%$, sugar $-67 \mathrm{mg} \%$, CSF PCR for tuberculosis and culture were both negative. Anti-nuclear antibodies(ANA),anti double stranded deoxy-ribonucleic acid antibodies( anti ds DNA), extractable nuclear antigen( ENA),rheumatoid factor, C reactive protein, ELISA for human immune deficiency virus 1 and 2, thyroid profile (Free T3, Free T4, thyroid stimulating hormone), anti thyroid peroxidase (TPO) antibody were normal. Magnetic resonance angiography (MRA) of intracranial vessels and gadolinium contrast MRI of brain was normal. Patient improved with supportive therapy, but had another episode four days later. These investigations have made no advance towards a definite diagnosis, so a possibility of pseudo migraine with CSF lymphocytic pleocytosis was arrived at. Anti tubercular drugs were discontinued. The patient recovered completely with supportive treatment and continued to remain symptom free for next 6 month follow up.

\section{Discussion}

Headache with transient neurological deficits and CSF lymphocytosis is also known as "hemiplegic migraine with cerebrospinal fluid abnormalities"[1] and "psueudomigraine with transient neurological symptoms and lymphocytic pleocytosis"[4]. Diagnostic criteria have been laid down only recently[3]. The syndrome is a triad of transient focal neurological deficits, headache and lymphocytic pleocytosis.
The focal symptoms resemble migraine with a slow evolution over minutes to hours than sudden onset which is more characteristic of a TIA. HaNDL is a self limiting illness lasting from several hours to weeks with no reported recurrence as of date. It is mostly a disease of young adults (peak age of $14-39$ years), predominant amongst males (M: F- 2:1) and presenting with one or more episodes of neurological deficits. The focal neurological symptoms typically are followed by a moderate to severe bilateral throbbing headache, associated with nausea and vomiting in approximately $50 \%$ of patients [4]. Acute confusional state as the core picture of HaNDL is extremely rare, and only 4 cases had been reported in literature[5-8]. We illustrate here, such a patient who in addition to acute confusional state has meningism without any sensorimotor prodromes.

A lumbar puncture is essential for diagnosis. Lymphocytic pleocytosis is always present in the range 199 cells / cu. mm $\pm 174[4]$. Elevated CSF opening pressure and elevated CSF protein are common. CSF cultures and serology are normal[1-10]. Exclusion of infectious etiologies by CSF analysis is of utmost importance to rule out CNS infections. Focal slowing is the most frequently encountered EEG abnormality in HaNDL[4].

The pathophysiology of HaNDL is unclear. The latest hypothesis suggests auto antibodies against cerebral vasculature, which are induced by viral infection[4]. Appropriate and timely clinical recognition helps favorable prognostication as well as reassuring the patients and their caregiver.

\section{Conclusion}

Acute psychosis and meningism can therefore be the major neurologic prodrome of HaNDL, which presents as global cortical dysfunction during its ictal phase and without any sensorimotor prodromes as is classically described. It is important for physicians to differentiate HaNDL from other potentially fatal neurologic disorders. Detailed review of history and neurologic assessment, prompt neuroimaging and CSF study are useful in excluding the potential life threatening neurologic illness, thereby enabling them to institute the necessary treatment. Such expertise to delineate HaNDL would prevent the patient from being subjected to un-necessary intervention like thrombolysis, long term pharmacological treatment like antitubercular drugs and the complications thereof.

\section{References}

[1] Maarten G. Lansberg, Andrew R. Woofenden, Alexander M. Norbash, Don B. Smith and Gregory B. Albers. Headache with neurological deficits and CSF lymphocytosis: A transient ischemic attack mimic. Journal of Stroke and Cerebrovascular Diseases.1999:8: pp 42 - 44.

[2] Bartleson JD, Swanson JN, Whisnant JP. A migrainous syndrome with cerebrospinal fluid pleocytosis. Neurology 1981;31: $1257-1262$. 
[3] Berg MJ, Williams LS. The transient syndrome of headache with neurologic deficits and cerebrospinal fluid lymphocytosis. Neurology 1995; 45: 1648 - 1654.

[4] Gomez - Aranda F, Carradillas F, Marti - Masso JF, et al. Pseudomigraine with temporary neurological symptoms and lymphocytic pleocytosis. A report of 50 cases. Brain 1997; 120: 1105 - 1113 .

[5] Bo-Lin Ho, Chiou-: Lian Lai, Chung-Yao Hsu. Acute confusion in headache with neurologic deficits and cerebrospinal fluid lymphocytosis syndrome. Am J Emerg Med. 2012 Nov;30 (9):2078.e7-8.

[6] Mateo I, Pinedo A, Gomez - Beldarrain M, et al. Acute confusional state secondary to transient headache and neurological deficits with cerebrospinal fluid lymphocytosis.
Neurologia 2004; 19: $763-765$.

[7] Giorgetti A, Mariani G, Patruno GM, et al. The transient syndrome of headache with neurological deficits, cerebrospinal fluid pleocytosis and acute confusional state: a case report. J Headache Pain 2005; 6: 476.

[8] Parissis D, Ionnidis P, Balamoutsos G, et al. Confusional state in syndrome of HaNDL. Headache 2011; 51: $1285-$ 1288.

[9] Kremenitzer M, Golden GS. Letter. Hemiplegic migraine: Cerebrospinal fluid abnormalities. J Paediatr 1974; 85: 139.

[10] Schraeder PL, Burns KA. Hemiplegic migraine associated with an aseptic meningeal reaction. Arch Neurol 1980;37: $377-379$. 